\title{
New easternmost and southernmost records of Pseudoboa coronata Schneider, 1801 (Serpentes: Dipsadidae: Pseudoboini), with a distribution map
}

\author{
Henrique Caldeira Costa ${ }^{1^{*}}$, Giselle Agostini Cotta ${ }^{2}$ and Ross D. MacCulloch ${ }^{3}$ \\ 1 Universidade Federal de Minas Gerais, Instituto de Ciências Biológicas, Departamento de Zoologia, Laboratório de Herpetologia. \\ Avenida Antônio Carlos 6627, Pampulha, 31270-901. Belo Horizonte, MG, Brazil \\ 2 Fundação Ezequiel Dias, Unidade de Coleções Científicas e Popularização da Ciência, CEP 30510-010, Gameleira, Belo Horizonte, MG, Brazil \\ 3 Department of Natural History, Royal Ontario Museum, 100 Queens Park, Toronto, Ontario M5S 2C6, Canada \\ * Corresponding author. E-mail: ccostah@gmail.com
}

\begin{abstract}
The snake species Pseudoboa coronata has wide distribution from central Brazil to coastal Venezuela and the Guianas, eastern Bolivia, Colombia, Ecuador, and Peru. In this note, the known distribution range of $P$. coronata is extended eastward to the border between the states of Bahia and Pernambuco, and southward to Cachoeira Alta, state of Goiás, both in Brazil. Considering the limited biogeographic information for most taxa, reports like this add significant data for future studies on biogeography, taxonomy, and conservation biology.
\end{abstract}

Key words: Amazon Scarlet Snake, biogeography, Caatinga, Cerrado, distribution extension

Pseudoboini Bailey, 1967 is a monophyletic clade of Neotropical snakes containing the genera Boiruna, Clelia, Drepanoides, Mussurana, Oxyrhopus, Paraphimophis, Phimophis, Pseudoboa, Rhachidelus, Rodriguesophis, and Siphlophis (Grazziotin et al. 2012). Its type genus, Pseudoboa Schneider, 1801 currently has six recognized species: Pseudoboa coronata Schneider, 1801; Pseudoboa haasi (Boettger, 1905); Pseudoboa martinsi Zaher, Oliveira \& Franco, 2008; Pseudoboa neuwiedii (Duméril, Bibron \& Duméril, 1854); Pseudoboa nigra (Duméril, Bibron \& Duméril, 1854); and Pseudoboa serrana Morato, MouraLeite, Prudente \& Bérnils, 1995 (see Gaiarsa et al. 2013 for a summary of geographic distribution and natural history of Pseudoboini).

Pseudoboa coronata differs from its congeners by having 17 dorsal scale rows and uniform reddish or reddish brown dorsum in both juveniles and adults (Zaher et al. 2008). This South American species ranges from central Brazil through the Amazon basin to coastal Venezuela and the Guianas, as well as cis-Andean Colombia, Ecuador, Peru, and Bolivia (Gaiarsa et al. 2013). In Brazil it is recorded from Cerrado areas in the states of Goiás and Tocantins, and from Amazon Forest in Acre, Amazonas, Maranhão, Mato Grosso, Pará, Rondônia, and Roraima (e.g., Silva Jr. et al. 2005; Zaher et al. 2008; Bernarde et al. 2013). Despite its wide distribution range, $P$. coronata is considered to be rare, at least in some Amazonian areas (Cunha and Nascimento 1993). Here we present an eastward and southward extension of the currently known range of $P$. coronata, as well as a literature review and updated map of distribution records.

The range extensions are based on specimens housed in the snake collection of Fundação Ezequiel Dias (FUNED), in Belo Horizonte, state of Minas Gerais, Brazil (Resende and Cotta 2013). In 2011, while examining the specimens from FUNED's collection, we identified two Pseudoboa coronata: FUNED 213 (Figure $1 \mathrm{~A}$ and $1 \mathrm{~B}$ ), from the Usina Hidrelétrica Luiz Gonzaga (a hydroelectric power plant, previously called Itaparica), located in the São Francisco river on the boundary of the states of Bahia (at Glória, Rodelas, and Abaré counties) and Pernambuco (at Petrolândia, Floresta, Itacuruba, and Belém do São Francisco counties); and FUNED 902 (Figure $1 C$ and $1 D$ ), from Cachoeira Alta, Goiás.

The record from Luiz Gonzaga power plant is the first of $P$. coronata for the Caatinga ecoregion (Desert and Xeric Shrublands biome [Olson et al. 2001]), and also its new easternmost record (previously Nova Vida, $B R-316,25 \mathrm{~km}$ do Gurupi, state of Maranhão, Brazil) (Zaher et al. 2008). The record from Cachoeira Alta is the second record for Goiás and the southernmost record of $P$. coronata (previously in Santa Cruz de la Sierra, Bolivia [Fugler and Cabot 1995]). Morphological data of these two specimens are presented in Table 1. Specimens were identified with the aid of the dichotomous key and color patterns in Zaher et al. (2008). 

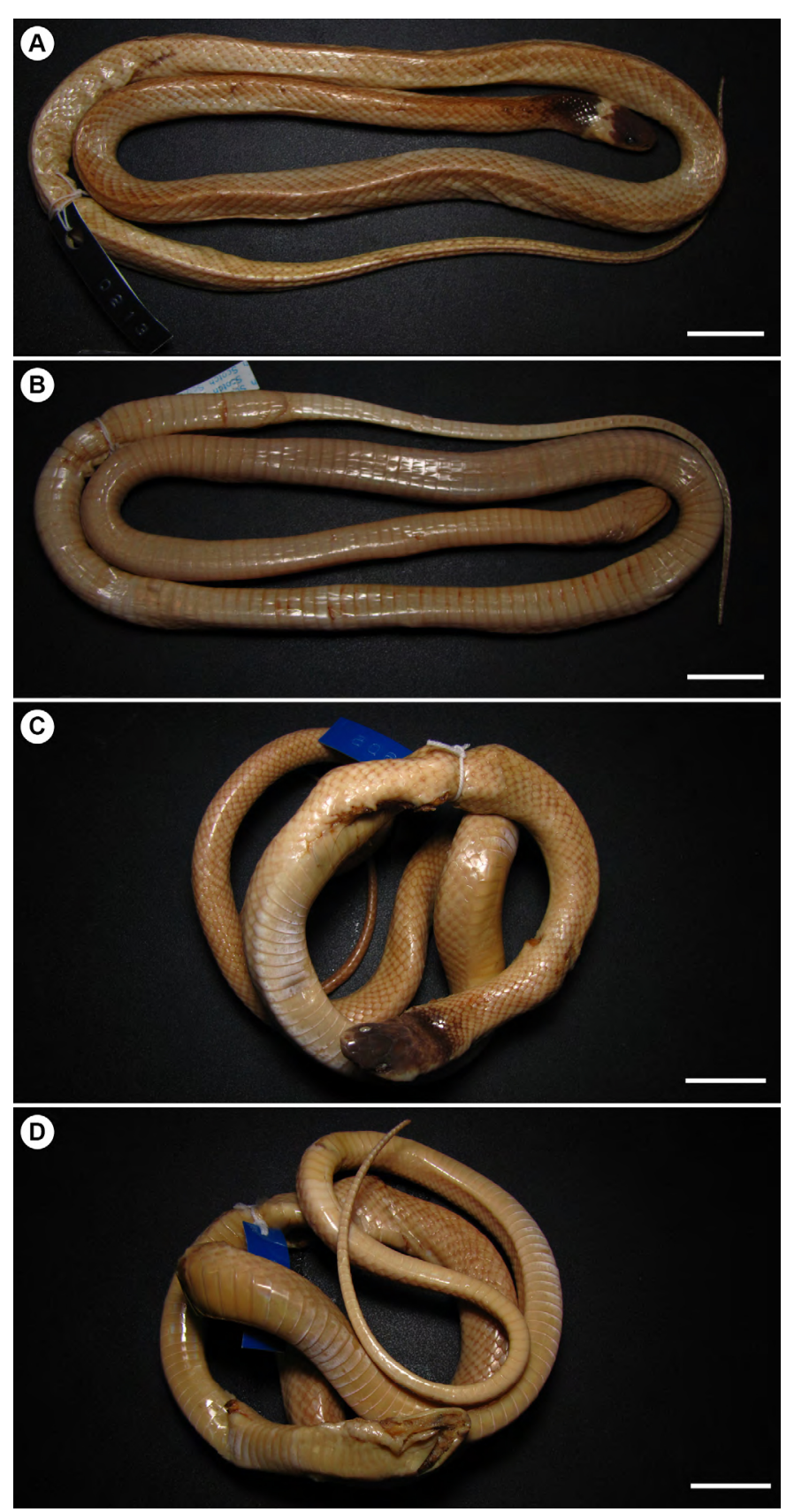

Figure 1. Specimens of Pseudoboa coronata used in this study. A and B: FUNED 213 (Luiz Gonzaga Hydroelectric Power Plant, Bahia/Pernambuco); C and D: FUNED 902 (Cachoeira Alta, Goiás). Scale bars $=20 \mathrm{~mm}$. Photos by H.C. Costa.

An updated map of distribution records of Pseudoboa coronata is presented in Figure 2, based on the new specimens and literature sources. Details about locality records are presented in Table 2. Some references that mention the occurrence of $P$. coronata with poor locality data were not used (i.e., Boulenger 1896; Avila-Pires 2005; Embert 2007; Rivas et al. 2012). When possible, coordinates were inferred from original mapped points (i.e., Chippaux 1986; Pérez-Santos and Moreno 1988; Starace 1998), although not all of them are associated with a voucher specimen in the original reference (e.g., some records from PérezSantos and Moreno 1988). Fugler and Walls (1978) cite an
Table 1. Morphological data of two specimens of Pseudoboa coronata from FUNED's snake collection: FUNED 213 (Usina Hidrelétrica Luiz Gonzaga, Bahia-Pernambuco border) and FUNED 902 (Cachoeira Alta, Goiás). SL+O $=$ supralabials contacting the eye; $\mathrm{IL}+\mathrm{C} 1=$ infralabials contacting the first pair of chinshields; IL+C2 = infralabials contacting the second pair of chinshields.

\begin{tabular}{lll}
\hline & FUNED 213 & FUNED 902 \\
\hline Snout-vent length & $644 \mathrm{~mm}$ & $491 \mathrm{~mm}$ \\
Caudal length & $179 \mathrm{~mm}$ & $173 \mathrm{~mm}$ \\
Sex & Female & Male \\
Dorsals & $17-17-17$ & $18-17-17$ \\
Apical pits & 2 & 2 \\
Ventrals & 189 & 180 \\
Subcaudals & 72 & 94 \\
Anal plate & entire & entire \\
Supralabials & 7 & 7 \\
SL+O & iii-iv & iii-iv \\
Infralabials & 7 & 8 \\
IL+C1 & i-iv & i-v \\
IL+C2 & iv & v \\
Nasal & divided & divided \\
Temporals & $1+2+2$ & $2+2+2$ \\
Preocular & 1 & 1 \\
Postocular & 2 & 2 \\
\hline
\end{tabular}

unlabeled specimen originally deposited at the University of North Carolina at Wilmington (UNC-W) in USA, collected at Mendez, in the Ecuadorian province of Morona Santiago, with a dorsal color pattern (dark brown with narrow cream bands) different from that usually found in P. coronata. The referred specimen was not found in the UNC-W collections (Alex Vance, pers. comm.), making it impossible to confirm its identity and, therefore, its record was not considered. The other five Ecuadorian specimens cited by Fugler and Walls (1978) are deposited at the United States National Museum, Smithsonian Institution. We were able to examine photographs of those specimens, but one of them (USNM 204156 from Macuma, Morona Santiago, Ecuador) has a brownishgray dorsum in preservative, suggesting it was not red in life. Since a careful analysis of this specimen was not possible we were not sufficiently confident to include it in our database. Those specimens cited by Fugler and Walls (1978) and Cole et al. (2013) were the only specimens cited in the literature that were examined by us.

It is worth noting that some old records of Pseudoboa coronata were mistakenly based on other species: e.g., Wied (1824) (P. nigra), Jan and Sordelli (1870) (plate V, figures 3 and 4: probably $P$. neuwiedii; plate VI, figure 2: P. nigra, based on Wied [1824]), and Boulenger (1896) (records from Rio de Janeiro and Petrópolis are of P. serrana, according to Morato et al. 1995).

Due to the existence of many biodiversity shortcomings in biological sciences, especially the Wallacean shortfall (limited biogeographic information for most taxa; Whittaker et al. 2005; Cardoso et al. 2011), reports 


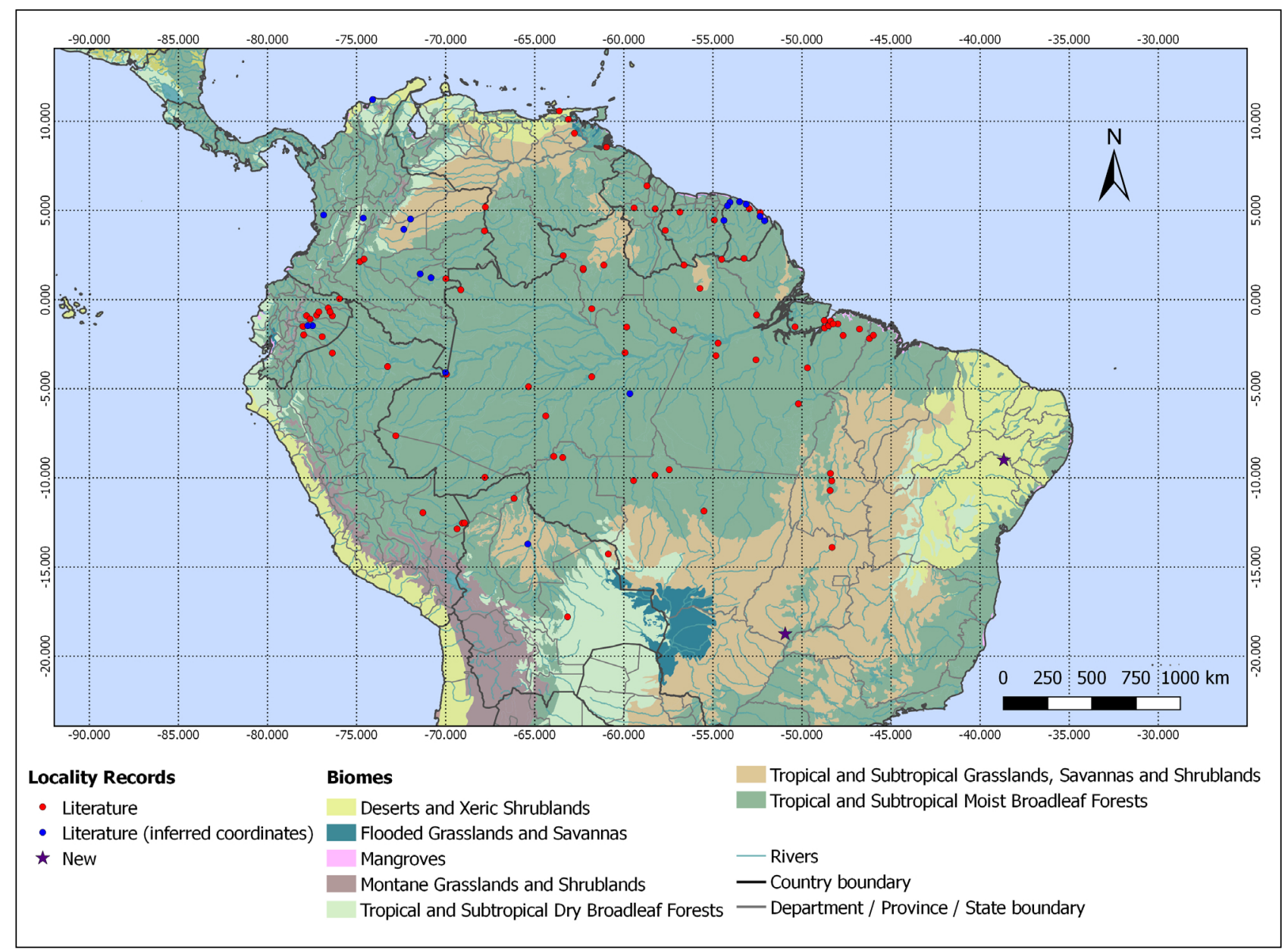

Figure 2. Known records of Pseudoboa coronata. Red dots: locality records from which geographic coordinates were given by the original source or had been taken from Costa et al. (2013), Paynter and Taylor (1991), Paynter (1993), IBGE (2011) or Google Earth ${ }^{\circ}$. Blue circles: locality records represented as unnamed mapped points or cited as river names in the original source, from which geographic coordinates had to be inferred. Purple stars: new records.

of range extensions add significant data for future studies on biogeography, taxonomy, and conservation biology. Furthermore, the wide range of Pseudoboa coronata, its occurrences in a variety of habitats, and the absence of a careful review of its taxonomy support the hypothesis that more than one species could have been treated under the same name.

\section{ACKNOWLEDGEMENTS}

The review of specimens from FUNED's snake collection was funded by Fundação de Amparo à Pesquisa no Estado de Minas Gerais (FAPEMIG), through the Programa Biota Minas. We thank Flávia C. Resende for measuring the specimens. Marinus S. Hoogmoed kindly provided locality data of Surinam specimens. We are also grateful to Omar Torres-Carvajal and the Museo de Zoología QCAZ (Pontificia Universidad Católica del Ecuador) for allowing the use of data from ReptiliaWebEcuador (http://zoologia.puce.edu.ec/Vertebrados/Reptiles/ ReptilesEcuador/Default.aspx); Alex Vance for information about specimens housed at UNC-W collections; James Poindexter for photographs of specimens from USNM;
Caryne Braga for her useful tips about mapping. Davi L. Pantoja (subject editor) and Vinícius A. São-Pedro (reviewer) provided useful comments and suggestions.

\section{LITERATURE CITED}

Avila-Pires, T.C.S. 2005. Reptiles; pp. 25-40, in: T. Hollowell and R.P. Reynolds (eds.). Checklist of the Terrestrial Vertebrates of the Guiana Shield. Bulletin of the Biological Society of Washington 13: 98 pp. doi: 10.2988/0097-0298(2005)13[25:R]2.0.CO;2

Avila-Pires, T.C.S., L.J. Vitt, S.S. Sartorius and P.A. Zani. 2009. Squamata (Reptilia) from four sites in southern Amazonia, with a biogeographic analysis of Amazonian lizards. Boletim do Museu Paraense Emílio Goeldi, Ciências Naturais 4(2): 99-118. http://www.museu-goeldi.br/editora/bn/artigos/cnv4n2_2009/ squamata(avilapires).pdf

Avila-Pires, T.C.S., M.S. Hoogmoed and W.A. Rocha. 2010. Notes on the Vertebrates of northern Pará, Brazil: A forgotten part of the Guianan Region, I. Herpetofauna. Boletim do Museu Paraense Emílio Goeldi. Ciências Naturais 5(1): 13-112. http://www.museugoeldi.br/editora/bn/artigos/cnv5n1_2010/notes(pires).pdf

Beebe, W. 1946. Field notes on the snakes of Kartabo, British Guiana, and Caripito, Venezuela. Zoologica 31(1): 11-52.

Bernarde, P.S., S. Albuquerque, D.B. Miranda and L.C.B. Turci. 2013. Herpetofauna da floresta do baixo rio Moa em Cruzeiro do Sul, Acre - Brasil. Biota Neotropica 13(1): 220-244. doi: 10.1590/ S1676-06032013000100023 
Table 2. Locality records of Pseudoboa coronata. Geographic coordinates not provided in the original source were taken from Costa et al. (2013), Paynter and Taylor (1991), Paynter (1993), IBGE (2011), Google Earth ${ }^{\circ}$. An asterisk $\left(^{*}\right)$ indicates geographic coordinates inferred for locality records represented as unnamed mapped points or cited only as river names in the original source.

\begin{tabular}{|c|c|c|c|c|c|}
\hline Country & Province/State & Municipality, Locality & Lat. & Long. & Source \\
\hline Bolivia & Beni & ${ }^{*}$ Rio Yacuma & $-13.71^{\circ}$ & $-65.39^{\circ}$ & Fugler and Cabot 1995 \\
\hline Bolivia & Beni & Tumi Chucua & $-11.15^{\circ}$ & $-66.16^{\circ}$ & Fugler and Cabot 1995 \\
\hline Bolivia & Santa Cruz & Parque Nacional Noel Kempff Mercado & $-14.27^{\circ}$ & $-60.87^{\circ}$ & Killeen and Schulenberg 1998 \\
\hline Bolivia & Santa Cruz & Santa Cruz de la Sierra & $-17.80^{\circ}$ & $-63.16^{\circ}$ & Fugler and Cabot 1995 \\
\hline Brazil & Acre & Cruzeiro do Sul, Floresta do Rio Moa & $-07.64^{\circ}$ & $-72.80^{\circ}$ & Bernarde et al. 2013 \\
\hline Brazil & Acre & Rio Branco & $-09.97^{\circ}$ & $-67.80^{\circ}$ & Silva et al. 2012 \\
\hline Brazil & Amazonas & Canutama & $-06.53^{\circ}$ & $-64.38^{\circ}$ & Zaher et al. 2008 \\
\hline Brazil & Amazonas & Manaus, Reserva Adolpho Ducke & $-02.98^{\circ}$ & $-59.93^{\circ}$ & Martins and Oliveira 1998 \\
\hline Brazil & Amazonas & Presidente Figueiredo, Usina Hidrelétrica (UHE) Balbina & $-01.54^{\circ}$ & $-59.84^{\circ}$ & Silva Jr. and Sites Jr. 1995 \\
\hline Brazil & Amazonas & Reserva de Desenvolvimento Sustentável Piaguçu-Purus & $-04.33^{\circ}$ & $-61.80^{\circ}$ & Waldez et al. 2013 \\
\hline Brazil & Amazonas & ${ }^{*}$ Rio Jaraqui & $-05.28^{\circ}$ & $-59.66^{\circ}$ & Zaher et al. 2008 \\
\hline Brazil & Amazonas & Uaupés, lauareté & $00.55^{\circ}$ & $-69.15^{\circ}$ & Hoge et al. 1973 \\
\hline Brazil & Bahia/Pernambuco & Usina Hidrelétrica (UHE) Luiz Gonzaga & $-09.00^{\circ}$ & $-38.67^{\circ}$ & this study \\
\hline Brazil & Goiás & Cachoeira Alta & $-18.76^{\circ}$ & $-50.94^{\circ}$ & this study \\
\hline Brazil & Goiás & Usina Hidrelétrica (UHE) Serra da Mesa / UHE Cana Brava & $-13.90^{\circ}$ & $-48.31^{\circ}$ & Silva Jr. et al. 2005 \\
\hline Brazil & Maranhão & Aldeia Araçu, Igarapé Gurupi-Una & $-02.18^{\circ}$ & $-46.21^{\circ}$ & Zaher et al. 2008 \\
\hline Brazil & Maranhão & Nova Vida, BR-316, 25 km do Gurupi & $-02.00^{\circ}$ & $-45.99^{\circ}$ & Cunha and Nascimento 1978, 1983 \\
\hline Brazil & Mato Grosso & Apiacás & $-09.54^{\circ}$ & $-57.45^{\circ}$ & Zaher et al. 2008 \\
\hline Brazil & Mato Grosso & Aripuanã & $-10.15^{\circ}$ & $-59.45^{\circ}$ & Zaher et al. 2008 \\
\hline Brazil & Mato Grosso & Cotriguaçu, Fazenda São Nicolau & $-09.85^{\circ}$ & $-58.25^{\circ}$ & Kawashita-Ribeiro et al. 2011 \\
\hline Brazil & Mato Grosso & Sinop & $-11.86^{\circ}$ & $-55.50^{\circ}$ & Zaher et al. 2008 \\
\hline Brazil & Pará & Agropecuária Treviso, Rio Curuá-Una & $-03.15^{\circ}$ & $-54.83^{\circ}$ & Avila-Pires et al. 2009 \\
\hline Brazil & Pará & Almeirim, Monte Dourado & $-00.86^{\circ}$ & $-52.55^{\circ}$ & Frota et al. 2005 \\
\hline Brazil & Pará & Ananindeua & $-01.37^{\circ}$ & $-48.38^{\circ}$ & Galatti and Yuki 1998 \\
\hline Brazil & Pará & $\begin{array}{l}\text { Barcarena, Area under influence of the Projeto Fauna, Refinaria } A B C \\
\text { - Rescue Area }\end{array}$ & $-01.59^{\circ}$ & $-48.73^{\circ}$ & Silva et al. 2011 \\
\hline Brazil & Pará & Belém & $-01.46^{\circ}$ & $-48.50^{\circ}$ & $\begin{array}{l}\text { Cunha and Nascimento 1983, } \\
\text { 1993; Zaher et al. } 2008\end{array}$ \\
\hline Brazil & Pará & Boa Vista, rio Apéu, próximo de Castanhal & $-01.36^{\circ}$ & $-47.99^{\circ}$ & Cunha and Nascimento 1978, 1983 \\
\hline Brazil & Pará & BR-316, km 224 & $-01.65^{\circ}$ & $-46.77^{\circ}$ & Cunha and Nascimento 1978, 1983 \\
\hline Brazil & Pará & Brasil Novo, Caverna Planaltina & $-03.38^{\circ}$ & $-52.58^{\circ}$ & Oliveira et al. 2013 \\
\hline Brazil & Pará & Breves, PA-159 km 18, comunidade Tancredo Neves & $-01.53^{\circ}$ & $-50.39^{\circ}$ & Zaher et al. 2008 \\
\hline Brazil & Pará & Cachoeira de Arari, Sé & $-01.17^{\circ}$ & $-48.75^{\circ}$ & Zaher et al. 2008 \\
\hline Brazil & Pará & Coari, Base Operacional Geólogo Pedro de Moura & $-04.89^{\circ}$ & $-65.35^{\circ}$ & Prudente et al. 2010 \\
\hline Brazil & Pará & Estação Ecológica do Grão Pará Centre & $00.63^{\circ}$ & $-55.73^{\circ}$ & Avila-Pires et al. 2010 \\
\hline Brazil & Pará & Floresta Estadual de Faro & $-01.71^{\circ}$ & $-57.21^{\circ}$ & Avila-Pires et al. 2010 \\
\hline Brazil & Pará & Floresta Nacional de Carajás & $-05.85^{\circ}$ & $-50.20^{\circ}$ & Maschio et al. 2012 \\
\hline Brazil & Pará & Ilha do Mosqueiro & $-01.20^{\circ}$ & $-48.39^{\circ}$ & Cunha and Nascimento 1978, 1983 \\
\hline Brazil & Pará & PA-252, km 16, partindo da BR-010 para o Acará & $-02.01^{\circ}$ & $-47.69^{\circ}$ & Cunha and Nascimento 1978,1983 \\
\hline Brazil & Pará & Santa Bárbara, Estrada do Mosqueiro & $-01.36^{\circ}$ & $-48.25^{\circ}$ & Cunha and Nascimento 1978, 1983 \\
\hline Brazil & Pará & Santarém & $-02.44^{\circ}$ & $-54.71^{\circ}$ & Frota et al. 2005 \\
\hline Brazil & Pará & Usina Hidrelétrica (UHE) Tucuruí & $-03.83^{\circ}$ & $-49.69^{\circ}$ & Zaher et al. 2008 \\
\hline Brazil & Rondônia & Porto Velho, Usina Hidrelétrica (UHE) Samuel & $-08.86^{\circ}$ & $-63.43^{\circ}$ & Silva Jr. 1993 \\
\hline Brazil & Rondônia & Porto Velho, Usina Hidrelétrica (UHE) Santo Antônio & $-08.80^{\circ}$ & $-63.93^{\circ}$ & Marçal et al. 2011 \\
\hline Brazil & Roraima ${ }^{\#}$ & $1 \mathrm{~km} \mathrm{~W}$ da BR-174 / 1-5 km N da BR-210 & $01.94^{\circ}$ & $-61.12^{\circ}$ & Zaher et al. 2008 \\
\hline Brazil & Roraima & Missão Catrimani & $01.68^{\circ}$ & $-62.28^{\circ}$ & Zaher et al. 2008 \\
\hline Brazil & Roraima & Rio Catrimani, Cachoeira do Cujubim & $01.75^{\circ}$ & $-62.28^{\circ}$ & Zaher et al. 2008 \\
\hline Brazil & Roraima & Santa Maria do Boiaçu & $-00.51^{\circ}$ & $-61.80^{\circ}$ & Zaher et al. 2008 \\
\hline Brazil & Roraima & Serra dos Surucucus & $02.47^{\circ}$ & $-63.40^{\circ}$ & Zaher et al. 2008 \\
\hline Brazil & Tocantins & Palmas & $-10.17^{\circ}$ & $-48.33^{\circ}$ & Marques et al. 2005 \\
\hline Brazil & Tocantins & Porto Nacional & $-10.71^{\circ}$ & $-48.42^{\circ}$ & Zaher et al. 2008 \\
\hline Brazil & Tocantins & Usina Hidrelétrica (UHE) Luís Eduardo Magalhães & $-09.75^{\circ}$ & $-48.40^{\circ}$ & Puorto and Barbarini 2004 \\
\hline Colombia & Amazonas & Leticia & $-04.19^{\circ}$ & $-69.95^{\circ}$ & Shreve 1947 \\
\hline Colombia & Amazonas & ${ }^{*}$ no further details & $-04.09^{\circ}$ & $-70.01^{\circ}$ & Pérez-Santos and Moreno 1988 \\
\hline Colombia & Caqueta & La Arenosa & $02.13^{\circ}$ & $-74.81^{\circ}$ & Cortes-Avila and Toledo 2013 \\
\hline Colombia & Casanare & ${ }^{*}$ no further details & $04.52^{\circ}$ & $-71.97^{\circ}$ & Pérez-Santos and Moreno 1988 \\
\hline Colombia & Chocó & ${ }^{*}$ no further details & $04.75^{\circ}$ & $-76.85^{\circ}$ & Pérez-Santos and Moreno 1988 \\
\hline Colombia & Cundinamarca & ${ }^{*}$ no further details & $04.57^{\circ}$ & $-74.62^{\circ}$ & Pérez-Santos and Moreno 1988 \\
\hline Colombia & Guainia & Estrella fluvial de Inirida & $03.85^{\circ}$ & $-67.82^{\circ}$ & Renjifo et al. 2009 \\
\hline Colombia & Madalena & ${ }^{*}$ no further details & $11.22^{\circ}$ & $-74.10^{\circ}$ & Pérez-Santos and Moreno 1988 \\
\hline
\end{tabular}


Table 2. Continued.

\begin{tabular}{|c|c|c|c|c|c|}
\hline Country & Province/State & Municipality, Locality & Lat. & Long. & Source \\
\hline Colombia & Meta & ${ }^{*}$ no further details & $03.94^{\circ}$ & $-72.35^{\circ}$ & Pérez-Santos and Moreno 1988 \\
\hline Colombia & Meta & San Juan del Losada & $02.27^{\circ}$ & $-74.58^{\circ}$ & Cortes-Avila and Toledo 2013 \\
\hline Colombia & Vaupés & *no further details & $01.44^{\circ}$ & $-71.43^{\circ}$ & Pérez-Santos and Moreno 1988 \\
\hline Colombia & Vaupés & ${ }^{*}$ no further details & $01.23^{\circ}$ & $-70.82^{\circ}$ & Pérez-Santos and Moreno 1988 \\
\hline Colombia & Vaupés & Timbó & $01.17^{\circ}$ & $-69.99^{\circ}$ & Kizirian and McDiarmid 1998 \\
\hline Ecuador & Morona Santiago & Chiguaza & $-01.98^{\circ}$ & $-77.97^{\circ}$ & Fugler and Walls 1978 \\
\hline Ecuador & Napo & Carretera a $1.5 \mathrm{~km}$ de Archidona vía a Quito & $-00.90^{\circ}$ & $-77.81^{\circ}$ & Pazmiño-Otamendi 2013 \\
\hline Ecuador & Napo & Estacion Biologica Jatun Sacha & $-01.09^{\circ}$ & $-77.61^{\circ}$ & Vigle 2008 \\
\hline Ecuador & Napo & Yachana Reserve & $-00.87^{\circ}$ & $-77.24^{\circ}$ & Whitworth and Beirne 2011 \\
\hline Ecuador & Orellana & La Belleza, Comunidad Bocana del Suno & $-00.69^{\circ}$ & $-77.12^{\circ}$ & Pazmiño-Otamendi 2013 \\
\hline Ecuador & Orellana & $\begin{array}{l}\text { Parque Nacional Yasuní, Bloque } 16 \text { de Repsol YPF, en carretera km } \\
57-58\end{array}$ & $-00.91^{\circ}$ & $-76.36^{\circ}$ & Pazmiño-Otamendi 2013 \\
\hline Ecuador & Orellana & Parque Nacional Yasuní, carretera Maxus 7.8 km & $-00.47^{\circ}$ & $-76.59^{\circ}$ & Pazmiño-Otamendi 2013 \\
\hline Ecuador & Orellana & Parque Nacional Yasuní, En pozo Capirón & $-00.69^{\circ}$ & $-76.49^{\circ}$ & Pazmiño-Otamendi 2013 \\
\hline Ecuador & Pastaza & Comunidad Santa Rosa & $-02.08^{\circ}$ & $-76.93^{\circ}$ & Pazmiño-Otamendi 2013 \\
\hline Ecuador & Pastaza & Puyo & $-01.50^{\circ}$ & $-78.01^{\circ}$ & Fugler and Walls 1978 \\
\hline Ecuador & Pastaza & *Río Liguino, tributary of Río Villano, upper Curaray & $-01.46^{\circ}$ & $-77.47^{\circ}$ & Fugler and Walls 1978 \\
\hline Ecuador & Pastaza & *Río Villano & $-01.46^{\circ}$ & $-77.74^{\circ}$ & Fugler and Walls 1978 \\
\hline Ecuador & Sucumbios & Santa Cecilia & $00.05^{\circ}$ & $-75.96^{\circ}$ & Duellman 1978 \\
\hline $\begin{array}{l}\text { French } \\
\text { Guiana }\end{array}$ & Camopi & Trois-Sauts & $02.31^{\circ}$ & $-53.25^{\circ}$ & $\begin{array}{l}\text { Gasc and Rodrigues 1980; } \\
\text { Chippaux 1986; Starace } 1998\end{array}$ \\
\hline $\begin{array}{l}\text { French } \\
\text { Guiana }\end{array}$ & Cayenne & Cayenne & $04.89^{\circ}$ & $-52.34^{\circ}$ & $\begin{array}{l}\text { Gasc and Rodrigues 1980; } \\
\text { Chippaux 1986; Starace } 1998\end{array}$ \\
\hline $\begin{array}{l}\text { French } \\
\text { Guiana }\end{array}$ & Cayenne & Kourou-Petit Saut & $05.10^{\circ}$ & $-52.96^{\circ}$ & Sheehy et al. 2014 \\
\hline $\begin{array}{l}\text { French } \\
\text { Guiana }\end{array}$ & Cayenne & ${ }^{*}$ no further details & $05.35^{\circ}$ & $-53.13^{\circ}$ & Chippaux 1986; Starace 1998 \\
\hline $\begin{array}{l}\text { French } \\
\text { Guiana }\end{array}$ & Cayenne & ${ }^{*}$ no further details & $04.67^{\circ}$ & $-52.35^{\circ}$ & Chippaux 1986; Starace 1998 \\
\hline $\begin{array}{l}\text { French } \\
\text { Guiana }\end{array}$ & Cayenne & ${ }^{*}$ no further details & $04.42^{\circ}$ & $-52.10^{\circ}$ & Starace 1998 \\
\hline $\begin{array}{l}\text { French } \\
\text { Guiana }\end{array}$ & Maripasoula & Mitaraca & $02.26^{\circ}$ & $-54.51^{\circ}$ & $\begin{array}{l}\text { Gasc and Rodrigues 1980; } \\
\text { Chippaux 1986; Starace } 1998\end{array}$ \\
\hline $\begin{array}{l}\text { French } \\
\text { Guiana }\end{array}$ & $\begin{array}{l}\text { St.-Laurent-du- } \\
\text { Maroni }\end{array}$ & Maroni (Maroni river?) & $04.44^{\circ}$ & $-54.38^{\circ}$ & $\begin{array}{l}\text { Gasc and Rodrigues 1980; Starace } \\
1998\end{array}$ \\
\hline $\begin{array}{l}\text { French } \\
\text { Guiana }\end{array}$ & $\begin{array}{l}\text { St.-Laurent-du- } \\
\text { Maroni }\end{array}$ & *no further details & $05.49^{\circ}$ & $-53.50^{\circ}$ & Starace 1998 \\
\hline $\begin{array}{l}\text { French } \\
\text { Guiana }\end{array}$ & $\begin{array}{l}\text { St.-Laurent-du- } \\
\text { Maroni }\end{array}$ & ${ }^{*}$ no further details & $05.46^{\circ}$ & $-54.04^{\circ}$ & Chippaux 1986; Starace 1998 \\
\hline $\begin{array}{l}\text { French } \\
\text { Guiana }\end{array}$ & $\begin{array}{l}\text { St.-Laurent-du- } \\
\text { Maroni }\end{array}$ & ${ }^{*}$ no further details & $05.26^{\circ}$ & $-54.18^{\circ}$ & Starace 1998 \\
\hline Guyana & Cuyuni-Mazaruni & Kartabo & $06.38^{\circ}$ & $-58.70^{\circ}$ & Beebe 1946 \\
\hline Guyana & Potaro-Siparuni & Kaieteur National Park & $05.14^{\circ}$ & $-59.42^{\circ}$ & Kok 2006 \\
\hline Guyana & $\begin{array}{l}\text { Upper Demerera- } \\
\text { Berbice }\end{array}$ & Berbice River & $05.09^{\circ}$ & $-58.24^{\circ}$ & Cole et al. 2013 \\
\hline Peru & Loreto & Andoas & $-03.00^{\circ}$ & $-76.36^{\circ}$ & Henle and Ehrl 1991 \\
\hline Peru & Loreto & Iquitos & $-03.76^{\circ}$ & $-73.26^{\circ}$ & Dixon and Soini 1977 \\
\hline Peru & Madre de Dios & Cuzco Amazonico & $-12.54^{\circ}$ & $-69.06^{\circ}$ & $\begin{array}{l}\text { Duellman and Salas 1991; Doan } \\
\text { and Arizabal 2002; Duellman } 2005\end{array}$ \\
\hline Peru & Madre de Dios & Eco Amazonia & $-12.53^{\circ}$ & $-68.94^{\circ}$ & Doan and Arizabal 2002 \\
\hline Peru & Madre de Dios & Pakitza, Manu National Park & $-11.95^{\circ}$ & $-71.28^{\circ}$ & Catenazzi et al. 2013 \\
\hline Peru & Madre de Dios & Sachavacayoc Centre & $-12.86^{\circ}$ & $-69.36^{\circ}$ & Doan and Arizabal 2002 \\
\hline Surinam & Brokopondo & Saracreek, $21 \mathrm{~km} \mathrm{~S}$ of Dam & $04.46^{\circ}$ & $-54.92^{\circ}$ & M.S. Hoogmoed, pers. comm. \\
\hline Surinam & Sipaliwini & Kutari Head & $01.94^{\circ}$ & $-56.62^{\circ}$ & M.S. Hoogmoed, pers. comm. \\
\hline Surinam & Sipaliwini & Road to Amotopo, km 212 & $03.88^{\circ}$ & $-57.67^{\circ}$ & M.S. Hoogmoed, pers. comm. \\
\hline Surinam & Sipaliwini & Upper Nickerie River & $04.91^{\circ}$ & $-56.85^{\circ}$ & M.S. Hoogmoed, pers. comm. \\
\hline Venezuela & Amazonas & Puerto Ayacucho/Venado (S of Samariapo) & $05.19^{\circ}$ & $-67.77^{\circ}$ & Gorzula and Senaris 1998 \\
\hline Venezuela & Delta Amacuro & Alrededores de Curiapo & $08.55^{\circ}$ & $-60.97^{\circ}$ & Molina et al. 2004 \\
\hline Venezuela & Monagas & Caripito & $10.11^{\circ}$ & $-63.11^{\circ}$ & $\begin{array}{l}\text { Beebe 1946; Roze 1966; Lasso et } \\
\text { al. } 2004\end{array}$ \\
\hline Venezuela & Monagas & Sabana de Maturín, cerca Uracoa & $09.33^{\circ}$ & $-62.77^{\circ}$ & Roze 1966 \\
\hline Venezuela & Sucre & Benitez, Parcelamiento Guaraúnos & $10.58^{\circ}$ & $-63.62^{\circ}$ & Rivas and La Marca 2001 \\
\hline
\end{tabular}

"Cited as "Rondônia" by Zaher et al. (2008). However, "Roraima” is the correct location, according to the MPEG collection register book (A.L.C. Prudente pers. comm.). 
Boulenger, G.A. 1896. Catalogue of the Snakes in the British Museum (Natural History). Volume III. London: Order of the Trustes. 727 pp. http://www.biodiversitylibrary.org/bibliography/29427

Cardoso, P., T.L. Erwin, P.A.V. Borges and T.R. New. 2011. The seven impediments in invertebrate conservation and how to overcome them. Biological Conservation 144(11): 2647-2655. doi: 10.1016/j. biocon.2011.07.024

Catenazzi, A., E. Lehr and R. von May. 2013. The amphibians and reptiles of Manu National Park and its buffer zone, Amazon basin and eastern slopes of the Andes, Peru. Biota Neotropica 13(4): 270-283. doi: 10.1590/S1676-06032013000400024

Chippaux, J.-P. 1986. Les Serpents de la Guyane Française. Paris: ORSTOM. 165 pp.

Cole, C.J., C.R. Townsend, R.P. Reynolds, R.D. MacCulloch and A. Lathrop. 2013. Amphibians and reptiles of Guyana, South America: Illustrated keys, annotated species accounts, and a biogeographic synopsis. Proceedings of the Biological Society of Washington 125(4): 317-620. doi: 10.2988/ooo6-324X-125.4.317

Cortes-Avila, L. and J.J. Toledo. 2013. Estudio de la diversidad de serpientes en areas de bosque perterbado y pastizal en San Vicente del Caguan (Caqueta), Colombia. Actualidades Biologicas 35(99): 185-197. http://www.scielo.org.co/pdf/acbi/v35n99/v35n99a5.pdf

Costa, H.C., M.R. Moura and R.N. Feio. 2013. Taxonomic revision of Drymoluber Amaral, 1930 (Serpentes: Colubridae). Zootaxa 3716(3): 349-394. doi: 10.11646/zootaxa.3716.3.3

Cunha, O.R. and F.P. Nascimento. 1978. Ofídios da Amazônia X. As cobras da região leste do Pará, Belém. Publicações Avulsas, Museu Paranaense Emílio Goeldi 31: 1-218.

Cunha, O.R. and F.P. Nascimento. 1983. Os Ofidios da Amazonia. XIX. As expécies de Oxyrhopus Wagler, com uma subespécie nova, e Pseudoboa Schneider, na Amazônia oriental e Maranhão (Ophidia: Colubridae). Boletim do Museu Paraense Emilio Goeldi, Zoologia 112: 1-42. http://repositorio.museu-goeldi.br/ jspui/handle/123456789/675

Cunha, O.R. and F.P. Nascimento. 1993. Os Ofidios da Amazonia. As cobras da região leste do Pará. Boletim do Museu Paraense Emilio Goeldi, Zoologia 9(1): 1-191.

Dixon, J.R. and P. Soini. 1977. The reptiles of the Upper Amazon basin, Iquitos region, Peru. II. Crocodilians, turtles and snakes. Milwaukee Public Museum, Contributions in Biology and Geology 12: 1-91.

Doan, T.M. and W. Arizabal A. 2002. Microgeographic variation in species composition of the herpetofaunal communities of Tambopata region, Peru. Biotropica 34(1): 101-117. doi: 10.1111/ j.1744-7429.2002.tboo246.x

Duellman, W.E. 1978. The biology of an equatorial herpetofauna in Amazonian Ecuador. Museum of Natural History, University of Kansas Miscellaneous Publication 65: 1-352. https://archive.org/ details/miscellaneouspub65univ

Duellman, W.E. 2005. Cuzco Amazonico: the lives of amphibians and reptiles in an Amazonian rainforest. Comstock Publishing, Ithaca, USA. 433 pp.

Duellman, W.E. and A.W. Salas. 1991. Annotated checklist of the amphibians and reptiles of Cuzco Amazonico, Peru. Occasional Papers of the Museum of Natural History, The University of Kansas 143: 1-13. http://www.biodiversitylibrary.org/ part/31706\#/summary

Embert, D. 2007. Distribution, diversity and conservation status of Bolivian reptiles. Ph.D. Dissertation. Bonn: FriedrichsWilhelms-Universitat. 429 pp. http://hss.ulb.uni-bonn.de/2008/ 1441/1441-engl.htm

Frota, J.G., A.P. Santos-Jr, H. Menezes-Chalkidis and A.G. Guedes. 2005. As serpentes da região do baixo rio Amazonas, oeste do estado do Pará, Brasil (Squamata). Biociências 13(2): 211-220. http://revistaseletronicas.pucrs.br/fo/ojs/index.php/fabio/ article/view/187
Fugler, C.M. and J. Cabot. 1995. Herpetologica Boliviana: Una lista comentada de las serpientes de Bolivia com datos sobre su distribucion. Ecología en Boliva 24: 41-85. http://bibliotecadigital. umsa.bo:8o8o/rddu/bitstream/123456789/1581/1/EEB-No24.PDF

Fugler, C.M. and A.B. Walls. 1978. Snakes of the Upano valley of Amazonian Ecuador. Journal of the Tennessee Academy of Science 53: 81-87. http://iweb.tntech.edu/sstedman/JTAS\%20 53-3.pdf

Gaiarsa, M.P., L.R.V. Alencar and M. Martins. 2013. Natural history of pseudoboine Snakes. Papéis Avulsos de Zoologia 53(19): 261-283. doi: 10.1590/So031-10492013001900001

Galatti, U. and R.N. Yuki. 1998. Pseudoboa coronata (False Coral Snake). Diet. Herpetological Review 29(1): 46.

Gasc, J.-P. and M.T. Rodrigues. 1980. Liste préliminaire des serpents de la Guyane française. Bulletin du Museum National d'Histoire Naturelle, Paris 2A2: 559-598.

Gorzula, S. and J.C. Señaris. 1998. Contribution to the herpetofauna of the Venezuelan Guayana I. A data base. Scientia Guayanae 8: 1-269.

Grazziotin, F.G., H. Zaher, R.W. Murphy, G. Scrocchi, M.A. Benavides, Y.P. Zhang, Y.P. and S.L. Bonatto. 2012. Molecular phylogeny of the New World Dipsadidae (Serpentes: Colubroidea): a reappraisal. Cladistics 1: 1-23. doi: 10.1111/j.1096-0031.2012.00393.x

Henle, K. and A. Ehrl. 1991. Zur reptilienfauna Perus nebst Beschreibung eines neuen Anolis (Iguanidae) und zweier neuer Schlangen (Colubridae). Bonnischer Zoologische Beiträge 42(2): 143-180. http://zfmk.de/BZB/1991/1991\%20Henle\%20K.\%20u.\%20Ehrl\%20 A.\%2op143.pdf

Hoge, A.R., N. Santos, C. Heitor, L.A. Lopes and I.M. Souza. 1973 “1972". Serpentes coletadas pelo Projeto Rondon VII em Iauaret'e, Brasil. Memórias do Instituto Butantan 36: 221-232.

IBGE (Instituto Brasileiro de Geografia e Estatística). 2011. Índice de nomes geográficos. Volume 1. Rio de Janeiro: IBGE. Unpaginated. ftp://geoftp.ibge.gov.br/documentos/cartografia/ indice_nomes_geograficos.pdf

Jan, G. and F. Sordelli. 1870. Iconographie générale des ophidiens. Trente-Quatrième Livraison. Paris: J.-B. Baillière et Fils. Unpaginated; 6 pl. http://www.biodiversitylibrary.org/item/24266

Kawashita-Ribeiro, R.A., J.P. Silva, A.F. Silva, L.A.G. Arruda, T. Mott and M.A. Carvalho. 2011. Os Répteis Escamosos (Reptilia, Squamata) da Fazenda São Nicolau, Cotriguaçu, Mato Grosso, Brasil, um Estudo Preliminar; pp. 145-167, in: D.J. Rodrigues, T.J. Izzo and L.D. Battirola (orgs.). Descobrindo a Amazônia Meridional: Biodiversidade da Fazenda São Nicolau. Cuiabá: Pau e Prosa Comunicação.

Killeen, T.J. and T.S. Schulenberg. 1998. A biological assessment of Parque Nacional Noel Kempff Mercado, Bolivia. RAP Working Papers 10. Washington, D.C.: Conservation International. 372 pp.

Kizirian, D.A. and R.W. McDiarmid. 1998. A New Species of Bachia (Squamata: Gymnophthalmidae) with Plesiomorphic Limb Morphology. Herpetologica 54(2): 245-253. http://www.jstor. org/discover/10.2307/3893430

Kok, P.J.R. 2006. A new snake of the genus Atractus Wagler, 1828 (Reptilia: Squamata: Colubridae) from Kaieteur National Park, Guyana, northeastern South America. Zootaxa 1378: 19-35. http://www.mapress.com/zootaxa/2006f/z01378po35f.pdf

Lasso, C., L.A. Alonso, A.L. Flores and G. Love. 2004. Rapid assessment of the biodiversity and social aspects of the aquatic ecosystems of the Orinoco Delta and the Gulf of Paria, Venezuela. RAP Bulletin of Biological Assessment 37. Washington, D.C.: Conservation International. $359 \mathrm{pp}$.

Marçal, A.S., I.B.S.R. Gomes and J.T. Coragem (org.). 2011. UHE Santo Antônio: Guia das espécies de fauna resgatadas. São Paulo: Scriba Comunicação Corporativa. 327 pp.

Marques, O.A.V., A. Eterovic, C. Strüssmann and I. Sazima. 2005. Serpentes do Pantanal: guia ilustrado. Ribeirão Preto: Holos 
Editora. $184 \mathrm{pp}$

Martins, M. and M.E. Oliveira. 1998. Natural history of snakes in forests of the Manaus region, Central Amazonia, Brazil. Herpetological Natural History 6: 78-150.

Maschio, G.F., U. Galatti, S. Neckel-Oliveira, M. Gordo and Y.O.C. Bitar. 2012. Répteis de Carajás; pp. 82-97, in: F.D. Martins, A.F. Castilho, J. Campos, F.M. Hatano and S.G. Rolim. (orgs.). Fauna da Floresta Nacional de Carajás: Estudos sobre Vertebrados Terrestres. São Paulo: Nitro Imagens. http://www.icmbio.gov.br/ portal/images/Carajas.pdf

Molina, C., J.C. Senaris and G. Rivas. "2003" [2004]. Los reptiles del delta del Orinoco, Venezuela. Memoria de la Fundacion La Salle de Ciencias Naturales 159-160: 235-264. http://www. fundacionlasalle.org.ve/userfiles/11-Los\%2oreptiles\%2odel\%20 Delta.pdf

Morato, S.A.A., J.C. Moura-Leite, A.L.C. Prudente and R.S. Bérnils. 1995. A new species of Pseudoboa Schneider, 1801 from southeastern Brazil (Serpentes: Colubridae: Xenodontinae: Pseudoboini). Biociências 32(2): 253-264.

Oliveira, E.A., E.J. Hernández Ruz and F.B. Barros. 2013. Herpetofauna de las proximidades de la Caverna Planaltina, Brasil Novo, Pará (Amazonia brasileña). Herpetotropicos 9(1-2): 55-68. http://erevistas.saber.ula.ve/index.php/herpetotropicos/ article/view/4487

Olson, D.M., E. Dinerstein, E.D. Wikramanayake, N.D. Burgess, G.V.N. Powell, E.C. Underwood, J.A. D'Amico, I. Itoua, H.E. Strand, J.C. Morrison, C.J. Loucks, T.F. Allnutt, T.H. Ricketts, Y. Kura, J.F. Lamoreux, W.W. Wettengel, P. Hedao and K.R. Kassem. 2001 Terrestrial ecoregions of the world: A new map of life on Earth. Bioscience 51(11): 933-938. doi: 10.1641/0006-3568(2001)051[0933:TEOTWA]2.0.CO;2

Pazmiño-Otamendi, G. 2013. Pseudoboa coronata; in: S.R. Ron, J.M. Guayasamin, M.H. Yanez-Muñoz, A. Merino-Viteri and D.A. AmphibiaWebEcuador. Version 2014.0. Museo de Zoología, Pontificia Universidad Católica del Ecuador. Accessed at http:// zoologia.puce.edu.ec/vertebrados/anfibios/FichaEspecie. aspx?Id=3117, 25 September 2014.

Paynter, R.A. 1993. Ornithological gazetteer of Ecuador. Second edition. Cambridge: Museum of Comparative Zoology. xi +247 pp. http://biodiversitylibrary.org/page/14519674

Paynter, R.A. and M.A. Traylor Jr. 1991. Ornithological gazetteer of Brazil (2 vols.). Cambridge: Museum of Comparative Zoology. viii + 789 pp. http://biodiversitylibrary.org/page/14529339

Pérez-Santos, C. and A.G. Moreno. 1988. Ofidios de Colombia. Museo Regionale di Scienze Naturali, Torino, Monographie VI, 517 pp.

Prudente, A.L.C., G.F. Maschio, M.C. Santos-Costa, D.T. Feitosa. 2010. Serpentes da Bacia Petrolífera de Urucu, Município de Coari, Amazonas, Brasil. Acta Amazonica 40(2): 381-386. doi: 10.1590/Soo44-59672010000200016

Puorto, G. and C.C. Barbarini. 2004. Coleção Herpetológica do Museu de Zoologia do Centro Universitário Luterano de Palmas - MZCEULP. Humanitas 4/6: 31-38.

Renjifo, J.M., C.A. Lasso and M.A. Morales-Betancourt. 2009. Herpetofauna de la Estrella Fluvial de Inírida (ríos Inírida, Guaviare, Atabapo y Orinoco), Orinoquia colombiana: lista preliminar de especies. Biota Colombiana 10(1-2): 171-178. http://www.bdigital. unal.edu.co/6645/1/Herpetofauna_de_la_Estrella_Fluvial_de_ In\% $C_{3} \% A D r i d a . p d f$

Resende, F.C. and G.A. Cotta. 2013. Ampliação da Coleção Científica de Serpentes da Fundação Ezequiel Dias. MG-Biota 5(4): 32-44. http://www.ief.mg.gov.br/images/stories/mg_biota/2014/ mg.biota\%20v.5\%20n.4.pdf

Rivas, G.A. and E. La Marca. 2001. Geographic distribution: Pseudoboa coronata (Crowned False Boa). Herpetological Review 32(2): 124.

Rivas, G.A., C.R. Molina, G.N. Ugueto, T.R. Barros, C.L. BarrioAmoros and P.J.R. Kok. 2012. Reptiles of Venezuela: an updated and commented checklist. Zootaxa 3211: 1-64. http://www. mapress.com/zootaxa/2012/2/zto3211po64.pdf

Roze, J.A. 1966. La taxonomia y zoogeografia de los ofidios en Venezuela. Caracas: Universidad Central de Venezuela. 359 pp.

Sheehy. C.M., M.H. Yánez-Muñoz, J.H. Valencia and E.N. Smith. 2014. A new species of Siphlophis (Serpentes: Dipsadidae: Xenodontinae) from the eastern Andean slopes of Ecuador. South American Journal of Herpetology 9(1): 30-45. doi: 10.2994/SAJH-D-12-00031.1

Shreve, B. 1947. On Colombian reptiles and amphibians collected by Dr. R.E. Schultes. Caldasia 4 (19): 311-316. http://www.revistas. unal.edu.co/index.php/cal/article/view/32664/32667

Silva, F.M., A.C. Menks, A.L.C. Prudente, J.C.L. Costa, A.E.M. Travassos and U. Galatti. 2011. Squamate Reptiles from municipality of Barcarena and surroundings, state of Pará, north of Brazil. Check List 7(3): 220-226. http://www.checklist.org.br/ getpdf?SL060-09

Silva, M.V., M.B. Souza and P.S. Bernarde. 2012 "2010". Riqueza e dieta de serpentes do Estado do Acre, Brasil. Revista Brasileira de Zoociências 12 (2): 165-176. http://zoociencias.uff.emnuvens. com.br/zoociencias/article/view/1583/1134

Silva Jr., N.J. 1993. The snakes from Samuel Hydroelectric Power Plant and vicinity, Rondônia, Brazil. Herpetological Natural History 1: 37-86.

Silva, Jr., N.J. and J.W. Sites Jr. 1995. Patterns of Diversity of Neotropical Squamate Reptile Species with Emphasis on the Brazilian Amazon and the Conservation Potential of Indigenous Reserves. Conservation Biology 9(4): 873-901. doi: 10.1046/j.1523-1739.1995.09040873.x

Silva Jr., N.J., H.L.R. Silva, M.T. Rodrigues, N.C. Valle, M.C. Costa, S.P. Castro, E.T. Linder, C. Johansson and J.W. Sites Jr. 2005. A fauna de vertebrados do alto rio Tocantins em áreas de usinas hidrelétricas. Estudos 32(1): 57-101.

Starace, F. 1998. Guide des serpents et amphisbènes de Guyane. Guadeloupe et Guyane: Ibis Rouge. 450 pp.

Vigle, G.O. 2008. The amphibians and reptiles of the Estacion Biologica Jatun Sacha in the lowland rainforest of Amazonian Ecuador: a 20-year record. Breviora 514: 1-30. doi: 10.3099/00069698-514.1.1

Waldez, F. M. Menin and R.C. Vogt. 2013. Diversidade de anfíbios e répteis Squamata na região do baixo rio Purus, Amazônia Central, Brasil. Biota Neotropica 13(1): 300-316. doi: 10.1590/ S1676-06032013000100029

Wied. M.P. 1824. Abbildungen Zur Naturgeschichte Brasiliens. Lief 7. Weimar: Landes-Industrie-Comptoir. Six plates, Unpaginated. http://www.biodiversitylibrary.org/item/109538

Whittaker, R.J., M.B. Araújo, P. Lepson, R.J. Ladle, J.E.M. Watson and K.J. Willis. 2005. Conservation biogeography: assessment and prospect. Diversity and Distributions 11(1): 3-23. doi: 10.1111/j.1366-9516.2005.00143.x

Whitworth, A. and C. Beirne. 2011. Reptiles of the Yachana Reserve. Exeter: Global Vision International. 127 pp. http://www.cadwizz. net/frogs/index.php?option=com_content\&view=article\&id=10 3\&Itemid $=74$

Zaher, H., M.E. Oliveira and F.L. Franco. 2008. A new, brightly colored species of Pseudoboa Schneider, 1801 from the Amazon Basin (Serpentes, Xenodontinae). Zootaxa 1674: 27-37. http:// www.mapress.com/zootaxa/2008/f/z01674p037f.pdf

Authors' contribution statement: All authors contributed to text writing. HCC and RDM have examined specimens and conducted bibliographic search.

Received: February 2014

Accepted: January 2015

Editorial responsibility: Davi Pantoja 